\title{
An Advanced Lyophilization Toward Intact Lipid Nanovesicles: Liquid-mediated Freezing With Cryoprotectant to Retain the Integrity of Lipid Nanovesicles
}

\author{
Eunhye Yang \\ Seoul National University \\ Hyunjong Yu \\ Seoul National University \\ SungHak Choi \\ Seoul National University \\ Kyung-Min Park \\ Wonkwang University \\ Ho-Sup Jung \\ Seoul National University \\ Pahn-Shick Chang ( $\sim$ pschang@snu.ac.kr) \\ Seoul National University
}

\section{Research Article}

Keywords: Liquid-mediated freezing (LMF), novel lyophilization method, lipid nanovesicles, cryoprotectant, integrity

Posted Date: September 7th, 2021

DOI: https://doi.org/10.21203/rs.3.rs-863913/v1

License: (c) (i) This work is licensed under a Creative Commons Attribution 4.0 International License. Read Full License 


\section{Abstract}

A novel lyophilization method using liquid-mediated freezing (LMF) with cryoprotectant (CPA) was designed to achieve intact lipid nanovesicles after lyophilization. During the freezing step, CPA prevented water supercooling, and the freezing rate was controlled by LMF. Regulating the freezing rate by various liquid media was a crucial determinant of membrane disruption, and isopropanol (freezing rate of $0.933^{\circ} \mathrm{C} / \mathrm{min}$ ) was the optimal medium for the LMF system. Lyophilized lipid nanovesicle using both LMF and CPA retained $92.9 \%$ of the core material and had uniform size distributions (Z-average diameter $=$ $135.5 \mathrm{~nm}$, polydispersity index $=0.074)$, similar to intact vesicles $(112.3 \mathrm{~nm}$ and 0.184 , respectively), after rehydration. Only lyophilized lipid nanovesicle using both LMF and CPA showed no changes in membrane fluidity and polarity. This lyophilization method can be applied to improve storage stability of lipid nanocarriers encapsulating drugs such as a mRNA vaccine, while retaining its original activity.

\section{Introduction}

Lipid nanovesicles, which are spherical particles formed from lipid bilayers enclosing discrete aqueous spaces, serve as nanocarriers of biologically and therapeutically active compounds for biodistribution to target sites ${ }^{1,2}$. Lipid nanovesicles can improve the pharmacokinetics and biodistribution of theragnostic compounds because of their inherent advantages, including high compound-loading efficiency, stability in biological environments, controllable release kinetics, and biocompatibility ${ }^{3,4}$. Hence, lipid nanovesicles have been used commercially as drug delivery systems, carriers for medical diagnostics, ${ }^{5,6}$ mRNA vaccine delivery systems with minimal toxicity, ${ }^{7,8}$ signal enhancers in analytical biochemistry, ${ }^{9}$ and solubilizers for antibiotics to kill pathogens ${ }^{10-15}$.

Lipid nanovesicles contain large amounts of unsaturated fatty acids, which can cause instability in the membrane structure depending on the external conditions, limiting their commercial application. Modification of the membrane surface, ${ }^{11,12}$ grafting polymers to the lipid head group, ${ }^{13}$ and solidification via lyophilization and spray drying have been employed to improve the physicochemical stability of lipid nanovesicles. Lyophilization is widely used for the solidification and storage of vesicle formulations as it prevents degradation, aggregation, or fusion of the nanovesicles by reducing interactions between the lipid membrane and water, thus improving stability ${ }^{14-16}$. Although lyophilization has several advantages over other solidification methods, lipid bilayer membranes can be damaged during lyophilization because of the physical stress caused by the formation of ice crystals during the freezing step ${ }^{16}$. Attempts to improve the integrity of lipid bilayer membranes by incorporating a cryoprotectant (CPA) in the lipid nanovesicle solution to support a frozen matrix have been unsuccessful in terms of maintaining intact nanovesicle size distributions and morphology. These results suggest that the use of CPA alone is insufficient to retain the integrity of membranes during lyophilization.

The freezing rate considerably affects membrane structure during lyophilization, which causes differences in ice crystal growth. Quick freezing (e.g., freezing rate of $\left.70^{\circ} \mathrm{C} / \mathrm{min}\right)$, in which small-volume 
suspensions are immersed in liquid nitrogen, reduces physical stress and metastable behavior via the formation of fine ice crystals ${ }^{17}$. However, extremely quick freezing creates an osmotic shock because of the difference in freezing point between internal and external aqueous phases in lipid nanovesicles ${ }^{18}$. Meanwhile, slow freezing (e.g., freezing rate of $0.5^{\circ} \mathrm{C} / \mathrm{min}$ ) reduces the osmotic pressure of the system, and water molecules can diffuse slowly across lipid bilayers ${ }^{19,20}$. However, the ice crystal size in lipid vesicles is larger with slow freezing compared with quick freezing; larger ice crystals cause physical damage to the lipid bilayer due to volume expansion of the ice ${ }^{21}$. Hence, precise control of the freezing rate is necessary to diminish disruption of lipid bilayer membranes by ice crystal formation.

Herein, we designed a novel lyophilization method using CPA and a liquid-mediated freezing (LMF) system to adjust the ice crystal growth of lipid nanovesicle solutions. Lipid nanovesicles were prepared from a soy-phosphatidylcholine lipid based on the microfluidic principle. Trehalose and sucrose were used as the CPA for the internal and external aqueous phases of the lipid nanovesicles, respectively. In the LMF system, organic solvents with a freezing point lower than $-75^{\circ} \mathrm{C}$ (deep freezing condition) were used as liquid media.

\section{Materials And Methods}

\section{Materials}

Soy phosphatidylcholine (soy-PC; lecithin from soybean) was purchased from llshin wells (Seoul, Korea). Cholesterol $(\mathrm{CH} ;>99 \%$ purity), trehalose dihydrate, sucrose, and calcein were purchased from SigmaAldrich Co. (St. Louis, MO, USA). 1,6-Diphenyl-1,3,5-hexatriene (DPH) and 6-dodecanoyl-N,N-dimethyl-2naphthylamine (Laurdan) were acquired from Molecular Probes (Eugene, OR, USA). Acetone, methanol, 1propanol, and isopropanol (IPA) were purchased from Daejung (Siheung, Gyeonggi-do, Korea). All other chemicals were obtained from Sigma-Aldrich Co. and used without further purification.

\section{Preparation of lipid nanovesicles with cryoprotectant}

Primary water-in-oil $\left(\mathrm{W}_{1} / 0\right)$ emulsions were prepared by emulsification. The $\mathrm{W}_{1}$ phase was prepared by dissolving trehalose $(15 \mathrm{mM})$, a cryoprotectant (CPA) for the internal aqueous phase of lipid nanovesicles, in distilled water. Calcein $(0.4 \mathrm{mM})$, a fluorescent probe typically used to be encapsulated within the internal aqueous phase of lipid nanovesicles, was added to the $\mathrm{W}_{1}$ phase. The oil phase comprised a mixture of soy-PC and $\mathrm{CH}$ to a molar ratio of 4:1 with a total lipid content of $3 \mathrm{mmol}$; soy-PC and $\mathrm{CH}$ were dissolved in ethyl acetate/ $n$-hexane $(4: 1, \mathrm{v} / \mathrm{v})$ and stirred for $5 \mathrm{~min}$ to form a solution containing the lipids. To form coarse inverted micelles, the mixture was homogenized for 5 min via sonication using a probe-type ultrasonicator (ULH-700S, Jeiotech, Korea). Then, to produce nano-size inverted micelles, the coarse micelle solution was homogenized in 10 cycles at $100 \mathrm{MPa}$ using a highpressure homogenizer (MN400BF, Micronox, Seongnam, Korea) primed with organic solvents. The nanosize inverted micelles were mixed with $600 \mathrm{~mL}$ of distilled water and $15 \mathrm{mmol}$ sucrose, which was used as the CPA for the external aqueous phase of the lipid nanovesicles. To prepare the nano-size double 
emulsion of water-in-oil-in-water $\left(\mathrm{W}_{1} / \mathrm{O} / \mathrm{W}_{2}\right)$, the coarse double emulsion solution was homogenized in five cycles at $30 \mathrm{MPa}$ using the high-pressure homogenizer primed with distilled water. After homogenization, the $\mathrm{W}_{1} / \mathrm{O} / \mathrm{W}_{2}$ emulsion was stirred at room temperature for $24 \mathrm{~h}$ to evaporate organic solvents between the lipid bilayers, producing lipid nanovesicles.

\section{Lyophilization of lipid nanovesicles}

For liquid-mediated freezing (LMF) system, a vessel containing liquid media was placed in a deep freezer at $-75^{\circ} \mathrm{C}$ for $4 \mathrm{~h}$, enabling the medium temperature to reach equilibrium at $-75^{\circ} \mathrm{C}$. Then, $30 \mathrm{~mL}$ of lipid nanovesicle solution in a $50 \mathrm{~mL}$ polystyrene tube was immersed in the pre-cooled liquid medium and frozen at $-75^{\circ} \mathrm{C}$ for $8 \mathrm{~h}$. Conventional freezing was performed by placing the lipid nanovesicle solution directly into the deep freezer for $8 \mathrm{~h}$. After freezing step, the freeze-drying process was performed only the primary drying using a freeze dryer (FD8512, Ilshin Lab Co., Ltd., Seoul, Korea) at $-75^{\circ} \mathrm{C}$ for $48 \mathrm{~h}$. The chamber pressure was maintained at $20 \mathrm{~Pa}$ during the freeze-drying process. The tubes containing the lyophilized lipid nanovesicles were immediately sealed and stored at $4^{\circ} \mathrm{C}$ until use.

\section{Characterization of lipid nanovesicles}

The lyophilized lipid nanovesicles $(100 \mathrm{mg}$ ) were rehydrated by adding $10 \mathrm{~mL}$ of distilled water and shaking gently using vortex mixer for $1 \mathrm{~min}$. Following rehydration, the mean diameters of the lipid nanovesicles were measured using a dynamic light scattering instrument (Zetasizer Nano ZS, Malvern, UK) with a submicron particle size analyzer.

For transmission electron microscopy (TEM), the lipid nanovesicles were stained with uranyl acetate as a negative staining reagent. Then, $10 \mu \mathrm{L}$ of lipid nanovesicle sample was dropped onto a formvar-coated carbon grid (200 mesh). After 1 min, the grid was loaded with $2 \%$ uranyl acetate solution (w/v) and incubated for $1 \mathrm{~min}$, and then washed with double-distilled water. The grid was dried at room temperature before the lipid nanovesicles were visualized by TEM ( $80 \mathrm{keV}$, JEOL Ltd., Tokyo, Japan).

\section{Evaluation of membrane fluidity and polarity}

The inner membrane fluidity of the lipid nanovesicles was evaluated as described previously ${ }^{22,23}$. The fluorescent probe DPH was added to the lipid nanovesicle suspension at a molar ratio of 250:1, lipid:DPH; the final concentrations of lipid and DPH were 100 and $0.4 \mu \mathrm{M}$, respectively. The solution was incubated at $30^{\circ} \mathrm{C}$ for $30 \mathrm{~min}$, then the fluorescence polarization of DPH (excitation $=360 \mathrm{~nm}$, emission $=430 \mathrm{~nm}$ ) was measured using a fluorescence spectrophotometer (FP-8500, JASCO, Tokyo, Japan). The sample was excited with vertically polarized light $(360 \mathrm{~nm})$, and the emission intensities perpendicular $\left(I_{\perp}\right)\left(0^{\circ}, 0^{\circ}\right)$ and parallel $\left(l_{\|}\right)\left(0^{\circ}, 90^{\circ}\right)$ to the excited light were measured at $430 \mathrm{~nm}$. The polarization (P) of DPH was calculated using the following equations:

$P=\left(I_{\|}-G I_{\perp}\right) /\left(I_{\|}+G I_{\perp}\right)$, 


$$
G=i_{\perp} / i_{\|}
$$

where $I_{\|}$and $I_{\llbracket}$ are the emission intensities parallel and perpendicular to the horizontally polarized light, respectively. $G$ is the correction factor, and $i_{\square}$ and $i_{\|}$are the emission intensities perpendicular and parallel to the vertically polarized light, respectively. The membrane fluidity was evaluated based on the reciprocal of polarization, $1 / P$.

The fluorescent probe Laurdan is sensitive to polarity, enabling the surface polarity of lipid membranes to be determined ${ }^{24-26}$. Laurdan emission spectra exhibit a red shift caused by dielectric relaxation. Thus, the emission spectra were calculated by obtaining the general polarization $\left(G P_{340}\right)$ for each emission wavelength as follows:

$G P_{340}=\left(I_{440}-I_{490}\right) /\left(I_{440}+I_{490}\right)$,

where $I_{440}$ and $I_{490}$ represent the emission intensities of Laurdan excited with $340 \mathrm{~nm}$ light. The total concentrations of lipid and Laurdan in the test solution were $200 \mu \mathrm{M}$ and $1 \mu \mathrm{M}$, respectively.

\section{Determination of encapsulation efficiency}

The encapsulation efficiency and core material retention of the lipid nanovesicles were determined fluorometrically using a previously developed method ${ }^{27}$. Briefly, $25 \mu \mathrm{L}$ of resuspended lipid nanovesicles (10 mg lipid/mL) were diluted to $500 \mu \mathrm{L}$ with $50 \mathrm{mM}$ MOPS buffer (pH 7.0) containing $110 \mathrm{mM}$ sodium chloride. Cobalt chloride $\left(\mathrm{CoCl}_{2}\right)$ was then added to quench the fluorescence of external calcein to eliminate background noise. Then, the lipid nanovesicles were lysed with detergent to determine the background fluorescence at zero encapsulated volume. Fluorescence intensity was measured before $\left(F_{\mathrm{b}}\right)$ and after $\left(F_{\mathrm{a}}\right)$ the addition of $5 \mu \mathrm{L}$ of $10 \mathrm{mM} \mathrm{CoCl}_{2}$, and before and after the addition of $25 \mu \mathrm{L} 10 \%$ Triton $\mathrm{X}-100\left(F_{\text {totq }}\right)$, at $490 \mathrm{~nm}$ excitation and $520 \mathrm{~nm}$ emission. Encapsulation efficiency was calculated as follows:

Encapsulation efficiency $(\%)=\left(F_{a}-F_{\text {totq }}\right) /\left(F_{b}-F_{\text {totq }}\right) \times 100$

\section{Temperature profiling}

The temperature profile was measured using a multi-channel thermometer (Dr.meter, Elitech, Taiwan). The temperature was measured every $1 \mathrm{~min}$. The temperature profiles of the lipid nanovesicle solutions during conventional freezing and LMF were measured by placing the thermometer sensor inside the sample at the center. The degree of supercooling was defined as the difference between the temperature at which ice crystals first form and the equilibrium freezing point of the solution ${ }^{28,29}$. The duration of ice crystal growth was defined as the length of time at which the temperature of the solution fell within the zone of ice crystal formation $\left(-5 \sim 0^{\circ} \mathrm{C}\right)^{30}$. 


\section{Results And Discussion}

\section{Freezing behavior of lipid nanovesicles}

As shown in Fig. 1a, the effects of LMF and CPA on ice crystal formation were assessed by plotting thermocouple temperature profiles of the lipid nanovesicle solution within the zone of ice crystal formation $\left(-5 \sim 0^{\circ} \mathrm{C}\right)$. The degree of supercooling and the freezing rate, which determine ice crystal formation, were measured in the lipid nanovesicle solution (Supplementary Fig. S1). The freezing process firstly involves ice nucleation, in which a minuscule crystalline lattice structure is formed. Once stable ice nuclei are formed, ice crystal growth proceeds by the addition of molecules to the interface ${ }^{31}$. Ice nucleation is affected by the supercooling of water, which is the retention of the liquid state below its equilibrium freezing point $\left(0^{\circ} \mathrm{C}\right)$. The freezing rate, the velocity to pass through the critical zone of ice crystal formation $\left(-5 \sim 0^{\circ} \mathrm{C}\right)$, determines the ice crystal size and distribution.

The degree of supercooling of the lipid nanovesicle solution was decreased by approximately half in the LMF system compared with the conventional freezing (CF) system (Fig. 1b). Meanwhile, the degree of supercooling of the solution was significantly lower with the addition of CPA than without CPA, indicating that supercooling was controlled primarily by CPA treatment. The presence of solutes such as CPA generally causes a decrease in the freezing point of solutions and promotes heterogeneous nucleation, which lead to a decrease in the degree of supercooling ${ }^{32,33}$. The combination of LMF and CPA completely suppressed supercooling of the lipid nanovesicle solution. The lowered degree of supercooling suggested that faster ice nucleation occurred in the solution, which contributed to a decrease in ice crystal size $\mathrm{e}^{34,35}$.

The freezing rate of the lipid nanovesicle solution in the $\mathrm{CF}$ system was $0.279^{\circ} \mathrm{C} / \mathrm{min}$, and it increased after adding CPA to the solution, which indicated that CPA treatment had little impact on ice crystal growth (Fig. 1C). On the other hand, the freezing rates of the lipid nanovesicle solution in the LMF system with and without CPA were 0.933 and $0.610^{\circ} \mathrm{C} / \mathrm{min}$, respectively. Compared with the CF system, the LMF system increased the freezing rate with and without CPA by 5.2- and 2.2-fold, respectively.

Generally, freezing at high rates reduces the time for ice crystals to grow in solution, thereby forming small and uniform ice crystals. Extremely quick freezing using liquid nitrogen can decrease the size of ice crystals more dramatically compared with freezing using the LMF system, but quick freezing also disrupts lipid bilayer membranes by creating high osmotic pressure ${ }^{36}$. Hence, freezing at a moderate rate (i.e., not too quick or too slow freezing) forms fine ice crystal and concurrently leads the appropriate diffusion from the aqueous phase of lipid nanovesicle ${ }^{37,38}$. These suggest that the LMF system regulating the freezing rate can minimize the membrane disruption from ice crystal formation.

\section{Effects of freezing rate on lipid nanovesicles}

Various organic solvents (e.g., acetone, $n$-propanol, ethanol, isopropanol, and methanol) as the liquid media were applied to the LMF system to control the freezing rate in detail. The temperature profiles of the lipid nanovesicle solutions in the LMF system using organic solvents showed that the freezing rate 
differed substantially depending on the liquid medium (Fig. 2a). The lowest freezing rate $\left(0.186^{\circ} \mathrm{C} / \mathrm{min}\right)$ was obtained using the $\mathrm{CF}$ system, whereas the highest freezing rate $\left(0.966^{\circ} \mathrm{C} / \mathrm{min}\right)$ was obtained using the LMF system with methanol as the liquid medium.

The freezing rate in the LMF system was significantly correlated with the specific heat capacity of the medium (Table S1). Linear regression indicated that the freezing rate increased proportionally with the specific heat capacity of the medium (Fig. 2b). Changes in the freezing rate were significantly associated with the removal of latent heat of fusion produced by the phase change of water from a liquid to crystalline form. These results suggest that as the specific heat capacity of the liquid medium increases, a higher driving force for heat transfer is provided to the sample solution, which increases the freezing rate accordingly. Furthermore, it is possible to precisely control the freezing rate by simply changing the liquid medium in the LMF system (Supplementary Fig. S2).

The effect of the freezing rate on the integrity of the lipid bilayer membrane was evaluated by measuring the size distribution of lipid nanovesicle after lyophilization (Fig. 2c). The intact lipid nanovesicle prior to lyophilization had a Z-average diameter $\left(\mathrm{D}_{\mathrm{z}}\right)$ of $112.3 \mathrm{~nm}$ and polydispersity index (PDI) of 0.184 , and transmission electron microscopy (TEM) images showed a monodispersed morphology of the lipid nanovesicles (Supplementary Fig. S3). Compared with the size distribution of intact lipid nanovesicles, changes in $\mathrm{D}_{\mathrm{z}}$ and PDI were observed in all of the lyophilized lipid nanovesicles. However, the changes in the size distributions of the lyophilized lipid nanovesicles differed significantly among the different freezing systems. The $D_{z}$ and PDI of lyophilized lipid nanovesicles showed the greatest changes in the CF and LMF systems with acetone and the smallest changes in the LMF system with isopropanol, suggesting that the change in size distribution decreased as the freezing rate increased (Supplementary Fig. S4). Nevertheless, lyophilized lipid nanovesicles in the LMF system with methanol, in which the freezing rate was highest, showed a greater increase in $D_{z}$ compared with the LMF system with isopropanol. These results suggest that too quick-freezing leads to disruption of the lipid bilayer membrane, and the membrane integrity of lipid nanovesicles can be maintained completely after lyophilization when the appropriate freezing rate is applied.

We assessed leakage of encapsulated material (fluorescent calcein in the internal aqueous phase) in lipid nanovesicles after lyophilization at different freezing rates (Fig. 2d). The highest leakage (25.32\%) was measured in lyophilized lipid nanovesicles in the CF system. Leakage from lyophilized lipid nanovesicles decreased as the freezing rate increased. But, the leakage in the LMF system using isopropanol showed the least leakage $(7.11 \%)$, consistent with the freezing rate-dependent changes in size distribution. These results demonstrate that minute changes in the freezing rate considerably affect the integrity of lipid bilayer membranes during freezing; thus, precise control of the freezing rate is crucial for maintaining the lipid nanovesicle structure. Isopropanol was selected as the optimal liquid medium in the LMF system for lyophilization of the lipid nanovesicles.

\section{Powderization and rehydration of lipid nanovesicles}


Powderization of lipid nanovesicles via lyophilization is a promising technology that can improve longterm stability and transportability for practical applications ${ }^{38,39}$. Lyophilized lipid nanovesicles must be reconstituted by adding a solution for rehydration prior to administration. The rehydration behavior of the lipid nanovesicle powder is important for product quality in terms of practical applications ${ }^{40}$. Hence, we examined the effects of LMF and CPA on the powderization and rehydration behavior of lipid nanovesicle powders.

As shown in Fig. 3a, the lipid nanovesicle powder lyophilized in the CF system formed a sticky agglomeration that did not completely disperse upon rehydration, regardless of CPA treatment. Lyophilization in the LMF system without CPA produced aggregated powder, which had low dispersibility following rehydration. By contrast, the powder produced by lyophilization in the LMF system with CPA had a fine and flour-like consistency and was dispersed completely in the solution without aggregation.

The size distributions of the lipid nanovesicle powders differed from those of intact lipid nanovesicles after rehydration, except for the powder produced by lyophilization using LMF with CPA (Fig. 3b). The lipid nanovesicle powder without CPA showed two size distribution peaks after rehydration, regardless of the freezing system. These results indicated that CPA prevented the collapse, fusion, or aggregation of lipid nanovesicles during lyophilization, during which the water molecules that interacted with the polar phosphate groups of lipid bilayers were displaced by CPAs such as saccharides. The surface of the frozen vesicles was covered by a concentrated aqueous saccharide solution or glassy solid, which prevented physical damage by ice crystals causing disruption of the lipid bilayer ${ }^{41}$. Meanwhile, the lipid nanovesicles lyophilized by CF (without LMF) with CPA showed 1.47- and 1.46-fold increases in $D_{z}$ and PDI after rehydration, respectively, compared with the intact lipid nanovesicles. Monodispersed size distributions of lipid nanovesicles similar to those of the intact vesicles were obtained only after lyophilization by LMF with CPA after rehydration. These results suggest that both the CPA treatment and LMF system are required to retain the integrity of lipid bilayer membranes during lyophilization and rehydration.

TEM images of the lipid nanovesicles lyophilized by CF without CPA and those lyophilized by LMF with CPA after rehydration are shown in Fig. 3c. Aggregations and structural disruptions were observed in the lipid nanovesicles lyophilized by CF in the absence of CPA. By contrast, the lipid nanovesicles lyophilized by LMF with CPA reverted to a spherical structure after rehydration, similar to intact lipid nanovesicles.

\section{Membrane fluidity and polarity of rehydrated lipid nanovesicles}

The effects of LMF and CPA on membrane integrity of lyophilized lipid nanovesicles were assessed by measuring membrane fluidity and polarity after rehydration. The membrane fluidity $(1 / P)$ values of rehydrated lipid nanovesicles after lyophilization with different freezing conditions (with or without LMF and CPA) were measured, and the results indicated membrane fluidity (Fig. 4a). The 1/Pvalues of lipid nanovesicles lyophilized in the LMF system, regardless of CPA treatment, were similar to those of the intact nanovesicles, suggesting that lyophilization in the LMF system did not cause considerable 
changes in the membrane fluidity of lipid nanovesicles. However, the $1 / P$ values of lipid nanovesicles lyophilized in the CF system (without LMF) in the presence or absence of CPA were higher than those of intact nanovesicles.

Membrane polarity of lyophilized lipid nanovesicles with different freezing conditions was determined using Laurdan, a fluorescence molecular probe. Laurdan shows specific emission peaks at 440 and 490 $\mathrm{nm}$ that originate from lipid bilayer membranes in ordered $\left(I_{0}, S_{0}\right)$ and disordered $\left(I_{\mathrm{d}}\right)$ phases, respectively. The lipid nanovesicles lyophilized under different freezing conditions showed different fluorescence spectra of Laurdan (Fig. 4b). An emission peak shift of Laurdan toward longer wavelengths (red shift) occurred in all lyophilized lipid nanovesicles, compared with the spectrum from intact lipid vesicles, but the peak shifts differed depending on the freezing conditions. Lyophilized lipid nanovesicles showed the lowest shift in the LMF system with CPA and the highest shift in the CF system without CPA. These results indicated that the freezing conditions determined the changes in the phase state of lipid membranes after lyophilization.

The membrane polarity $\left(G P_{340}\right)$ values of the lyophilized lipid nanovesicles were measured to identify the degree of hydration of the membrane surface (Fig. $4 \mathrm{c}$ ). The $G P_{340}$ value of the intact lipid nanovesicles was approximately 0.5 , whereas that of lyophilized lipid nanovesicles without CPA or LMF decreased significantly after rehydration. The decrease in the $G P_{340}$ value of lipid vesicles implied that the $I_{\mathrm{d}}$ phase increased, and the ordered $\left(S_{0}\right.$ or $\left.I_{0}\right)$ phase decreased, in the lipid membrane. Only lyophilized lipid nanovesicles treated with both CPA and LMF showed no change in the $G P_{340}$ value compared with intact vesicles. These results suggest that no fusion occurs, and the lipids remain in $I_{0}$ phase when lipid nanovesicles are lyophilized with both CPA and LMF. In other words, lyophilization with both CPA and LMF allows lipid nanovesicles to retain the trapped solute during rehydration since the vesicles do not undergo a phase transition.

In Fig. 4d, the membrane fluidity and polarity of lyophilized lipid nanovesicles are plotted as a Cartesian plot, in which the phase state of the lipid membranes can be classified based on the $G P_{340}$ and $1 / P$ values. The cross point of the $x$ and $y$ axes is the threshold point of the phase transition in the soyphosphatidylcholine lipid vesicle $\left(1 / P=6.0, G P_{340}=-0.2\right)^{42,43}$. Using Cartesian diagram analysis, lipid vesicles with lower fluidity $(1 / P<6)$ and higher polarity $\left(G P_{340}>-0.2\right)$ were plotted in the second quadrant, indicating that lipid membranes are in the $S_{0}$ or $I_{0}$ phase. The intact and lyophilized lipid nanovesicles in the LMF system existed in a solid-ordered $\left(S_{0}\right)$ phase with a lower fluidity and higher $G P_{340}$ value compared with lyophilized lipid nanovesicles in the CF system. Lyophilized lipid nanovesicles in the CF system, regardless of CPA treatment, were found near the boundary of the $S_{\mathrm{o}} / I_{\mathrm{o}}$ and $I_{\mathrm{d}}$ phases, indicating that they are in $I_{\mathrm{d}}$ phase partially mixed with the $S_{\mathrm{o}}$ or $I_{\mathrm{o}}$ phase. The plot showed that lipid membranes of intact and lyophilized lipid nanovesicles in the LMF system existed in the $S_{0}$ phase, with a lower fluidity and higher $G P_{340}$ value than those of the other nanovesicles. Meanwhile, the lyophilized 
lipid nanovesicles in the CF system were found near the boundary of the $S_{\mathrm{o}}$ and $I_{\mathrm{d}}$ phases, indicating that their lipid membranes are in a $I_{\mathrm{d}}$ phase partially mixed with the $S_{\mathrm{o}}$ or $I_{\mathrm{o}}$ phase.

These results suggest that the phase state of the lipid membrane was significantly altered during lyophilization in the CF system, which might be attributed to the formation of large and heterogeneous ice crystals, inducing membrane fusion of lipid nanovesicles. Nevertheless, only the lipid nanovesicles lyophilized with both CPA and LMF were found to be similar to intact nanovesicles in terms of the phase state of the membrane. These results suggest that membrane integrity is retained during lyophilization and completely reconstituted by rehydration only when both LMF and CPA are applied during the freezing step. The effects of CPA and LMF on the integrity of lipid nanovesicles during lyophilization and rehydration are presented in Fig. 5.

\section{Storage stability of lyophilized powder}

Lyophilization using CPA and LMF achieved optimal recovery of the lipid nanovesicle powder after longterm storage (Fig. 6). During storage at $4^{\circ} \mathrm{C}$ and $30^{\circ} \mathrm{C}$ for 30 days, the lipid nanovesicle powder retained its monodispersed distribution and colloidal stability unlike vesicles stored in solution (without lyophilization), which became unstable and aggregated after a short period ( 2 or 7 days). These results confirmed that this lyophilization method preserves intact lipid bilayer membrane structure for a long time. Rehydration is a complex process requiring multiple vortexing and heating steps to achieve complete reconstitution and solubilization of lipid nanovesicle powder ${ }^{17}$. By contrast, the proposed lyophilization methods in the LMF system using CPA can stabilize lipid bilayer membranes and prevent fusion, aggregation, and leakage. Furthermore, the process is comparatively simple; the lipid nanovesicle solution is frozen at $-75^{\circ} \mathrm{C}$ in a liquid medium (isopropanol) during the freezing step without the need for any complex device or process.

\section{Conclusion}

Lyophilization using LMF with CPA facilitates the powderization of lipid nanovesicles without agglomeration and retains the intrinsic physicochemical properties, including size distribution, structure, as well as membrane fluidity and polarity, after rehydration. We believe that the proposed method performs optimally in terms of processing and long-term storage of lipid nanovesicles, enhancing its applicability in the chemical, pharmaceutical, and food industries. Furthermore, this method can be applied to improve the original immunity and durability of mRNA-based vaccines with lipid nanocarriers, which are vulnerable to degradation during transportation and freezing storage.

\section{Declarations}

\section{Acknowledgements}

This study was carried out with the support of "R\&D Program for Forest Science Technology (Project No. 2019146C10-2121-AB02)" provided by Korea Forest Service (Korea Forestry Promotion Institute), and the 
National Research Foundation of Korea (NRF) Grant funded by the Ministry of Science and ICT for the First-Mover Program for Accelerating Disruptive Technology Development (NRF-2018M3C1B9069834).

\section{Author contributions}

E.Y. and H.Y. contributed equally to this work. E.Y. and H.Y. conducted experiments, analyzed data, and wrote the original manuscript. S.C. visualized and validated the results. K.M.P. had substantial contribution to conceptualization and methodology of the study. H.S.J. and P.S.C. served as cocorresponding authors, revised the manuscript, designed experiments, and supervised the study. All authors reviewed the manuscript.

\section{Competing interests}

The authors declare no competing interests.

\section{References}

1. Charrois, G. J. \& Allen, T. M. Drug release rate influences the pharmacokinetics, biodistribution, therapeutic activity, and toxicity of pegylated liposomal doxorubicin formulations in murine breast cancer. Biochim. Biophys. Acta 1663, 167-177, doi:10.1016/j.bbamem.2004.03.006 (2004).

2. Grimaldi, N. et al. Lipid-based nanovesicles for nanomedicine. Chem. Soc. Rev. 45, 6520-6545, doi:10.1039/c6cs00409a (2016).

3. Li, J. et al. Recent advancements in liposome-targeting strategies for the treatment of gliomas: A systematic review. ACS Appl. Bio Mater. 3, 5500-5528, doi:10.1021/acsabm.0c00705 (2020).

4. Chariou, P. L., Ortega-Rivera, O. A. \& Steinmetz, N. F. Nanocarriers for the delivery of medical, veterinary, and agricultural active ingredients. ACS Nano 14, 2678-2701, doi:10.1021/acsnano.0c00173 (2020).

5. Sun, Q. et al. Theranostic nanoplatform: Triple-modal imaging-guided synergistic cancer therapy based on liposome-conjugated mesoporous silica nanoparticles. ACS Appl. Mater. Inter. 10, 19631975, doi:10.1021/acsami.7b13651 (2018).

6. Zhang, C. et al. Multifunctional hybrid liposome as a theranostic platform for magnetic resonance imaging guided photothermal therapy. ACS Biomater. Sci. Eng. 4, 2597-2605, doi:10.1021/acsbiomaterials.8b00176 (2018).

7. Das, A., Asad, M., Sabur, A., Didwania, N. \& Ali, N. Monophosphoryl lipid a based cationic liposome facilitates vaccine induced expansion of polyfunctional $\mathrm{T}$ cell immune responses against visceral leishmaniasis. ACS Appl. Biomater. 1, 999-1018, doi:10.1021/acsabm.8b00184 (2018).

8. Chung, Y. H., Beiss, V., Fiering, S. N. \& Steinmetz, N. F. COVID-19 vaccine frontrunners and their nanotechnology design. ACS Nano 14, 12522-12537, doi:10.1021/acsnano.0c07197 (2020).

9. Mao, L., Yuan, R., Chai, Y., Zhuo, Y. \& Xiang, Y. Signal-enhancer molecules encapsulated liposome as a valuable sensing and amplification platform combining the aptasensor for ultrasensitive ECL 
immunoassay. Biosens. Bioelectron. 26, 4204-4208, doi:10.1016/j.bios.2011.02.035 (2011).

10. Lakshminarayanan, R., Ye, E., Young, D. J., Li, Z. \& Loh, X. J. Recent advances in the development of antimicrobial nanoparticles for combating resistant pathogens. Adv. Healthc. Mater. 7, 1701400, doi:10.1002/adhm.201701400 (2018).

11. Liu, W. et al. Physical-chemical stability and: In vitro digestibility of hybrid nanoparticles based on the layer-by-layer assembly of lactoferrin and BSA on liposomes. Food Funct. 8, 1688-1697, doi:10.1039/c7fo00308k (2017).

12. Lai, W.-F., Wong, W.-T. \& Rogach, A. L. Molecular design of layer-by-layer functionalized liposomes for oral drug delivery. ACS Appl. Mater. Inter. 12, 43341-43351, doi:10.1021/acsami.0c13504 (2020).

13. Labouta, H. I. et al. Surface-grafted polyethylene glycol conformation impacts the transport of PEGfunctionalized liposomes through a tumour extracellular matrix model. RSC Adv. 8, 7697-7708, doi:10.1039/c7ra13438j (2018).

14. Wessman, P., Edwards, K. \& Mahlin, D. Structural effects caused by spray- and freeze-drying of liposomes and bilayer disks. J. Pharm. Sci. 99, 2032-2048, doi: 10.1002/jps.21972 (2010).

15. Fonte, P. et al. Stability study perspective of the effect of freeze-drying using cryoprotectants on the structure of insulin loaded into PLGA nanoparticles. Biomacromolecules 15, 3753-3765, doi:10.1021/bm5010383 (2014).

16. Marín, D., Alemán, A., Montero, P. \& Gómez-Guillén, M. C. Encapsulation of food waste compounds in soy phosphatidylcholine liposomes: Effect of freeze-drying, storage stability and functional aptitude. J. Food Eng. 223, 132-143, doi:10.1016/j.jfoodeng.2017.12.009 (2018).

17. Franzé, S., Selmin, F., Samaritani, E., Minghetti, P. \& Cilurzo, F. Lyophilization of liposomal formulations: Still necessary, still challenging. Pharmaceutics 10, 139, doi:10.3390/pharmaceutics10030139 (2018).

18. Devireddy, R. V. The effect of extracellular ice and cryoprotective agents on the water permeability parameters of human sperm plasma membrane during freezing. Hum. Reprod. 15, 1125-1135, doi:10.1093/humrep/15.5.1125 (2000).

19. Li, D., Zhu, Z. \& Sun, D.-W. Effects of freezing on cell structure of fresh cellular food materials: A review. Trends Food Sci. Technol. 75, 46-55, doi:10.1016/j.tifs.2018.02.019 (2018).

20. Gurruchaga, H. et al. Advances in the slow freezing cryopreservation of microencapsulated cells. J. Control. Release 281, 119-138, doi:10.1016/j.jconrel.2018.05.016 (2018).

21. Deller, R. C., Vatish, M., Mitchell, D. A. \& Gibson, M. I. Synthetic polymers enable non-vitreous cellular cryopreservation by reducing ice crystal growth during thawing. Nat. Commun. $\mathbf{5}$, doi:10.1038/ncomms4244 (2014).

22. Hayashi, K. et al. Span 80 vesicles have a more fluid, flexible and "wet" surface than phospholipid liposomes. Colloid Surf. B-Biointerfaces 87, 28-35, doi:10.1016/j.colsurfb.2011.04.029 (2011).

23. Aloi, E., Rizzuti, B., Guzzi, R. \& Bartucci, R. Association of ibuprofen at the polar/apolar interface of lipid membranes. Arch. Biochem. Biophys. 654, 77-84, doi: 10.1016/j.abb.2018.07.013 (2018). 
24. Parasassi, T., De Stasio, G., Ravagnan, G., Rusch, R. M. \& Gratton, E. Quantitation of lipid phases in phospholipid vesicles by the generalized polarization of Laurdan fluorescence. Biophys. J. 60, 179189, doi:10.1016/S0006-3495(91)82041-0 (1991).

25. Parasassi, T. \& Gratton, E. Membrane lipid domains and dynamics as detected by Laurdan fluorescence. J. Fluoresc. 5, 59-69, doi:10.1007/BF00718783 (1995).

26. Parasassi, T., Krasnowska, E. K., Bagatolli, L. \& Gratton, E. Laurdan and Prodan as polarity-sensitive fluorescent membrane probes. J. Fluoresc. 8, 365-373, doi:10.1023/A:1020528716621 (1998).

27. Huang, S. L. \& MacDonald, R. C. Acoustically active liposomes for drug encapsulation and ultrasound-triggered release. Biochim. Biophys. Acta 1665, 134-141, doi:10.1016/j.bbamem.2004.07.003 (2004).

28. Liao, X., Krishnamurthy, R. \& Suryanarayanan, R. Influence of processing conditions on the physical state of mannitol-implications in freeze-drying. Pharm. Res. 24, 370-376, doi:10.1007/s11095-0069158-3 (2007).

29. Dou, M., Lu, C., Sun, Z. \& Rao, W. Natural cryoprotectants combinations of I-proline and trehalose for red blood cells cryopreservation. Cryobiology 91, 23-29, doi:10.1016/j.cryobiol.2019.11.002 (2019).

30. Luo, X. et al. Physicochemical changes of MTGase cross-linked surimi gels subjected to liquid nitrogen spray freezing. Int. J. Biol. Macromol. 160, 642-651, doi:10.1016/j.jbiomac.2020.05.249 (2020).

31. Assegehegn, G., Brito-De La Fuente, E., Franco, J. M. \& Gallegos, C. The importance of understanding the freezing step and its impact on freeze-drying process performance. J. Pharm. Sci. 108, 13781395, doi:10.1016/j.xphs.2018.11.039 (2019).

32. Kasper, J. C. \& Friess, W. The freezing step in lyophilization: Physico-chemical fundamentals, freezing methods and consequences on process performance and quality attributes of biopharmaceuticals. Eur. J. Pharm. Biopharm. 78, 248-263, doi:10.1016/j.ejpb.2011.03.010 (2011).

33. Petzold, G. \& Aguilera, J. M. Ice morphology: Fundamentals and technological applications in foods. Food Biophys. 4, 378-396, doi:10.1007/s11483-009-9136-5 (2009).

34. Searles, J. A., Carpenter, J. F. \& Randolph, T. W. The ice nucleation temperature determines the primary drying rate of lyophilization for samples frozen on a temperature-controlled shelf. J. Pharm. Sci. 90, 860-871, doi:10.1002/jps.1039 (2001).

35. Cochran, T. \& Nail, S. L. Ice nucleation temperature influences recovery of activity of a model protein after freeze drying. J. Pharm. Sci. 98, 3495-3498, doi:10.1002/jps.21815 (2009).

36. Izutsu, K.-I., Yomota, C. \& Kawanishi, T. Stabilization of liposomes in frozen solutions through control of osmotic flow and internal solution freezing by trehalose. J. Pharm. Sci. 100, 2935-2944, doi:10.1002/jps.22518 (2011).

37. Van Winden, E. C., Zhang, W. \& Crommelin, D. J. Effect of freezing rate on the stability of liposomes during freeze-drying and rehydration. Pharm. Res. 14, 1151-1160, doi:10.1023/a:1012142520912 (1997). 
38. Chen, C., Han, D., Cai, C. \& Tang, X. An overview of liposome lyophilization and its future potential. J. Control. Release 142, 299-311, doi:10.1016/j.jconrel.2009.10.024 (2010).

39. Ingvarsson, P. T., Yang, M., Nielsen, H. M., Rantanen, J. \& Foged, C. Stabilization of liposomes during drying. Expert Opin. Drug Deliv. 8, 375-388, doi:10.1517/17425247.2011.553219 (2011).

40. Sylvester, B. et al. Formulation optimization of freeze-dried long-circulating liposomes and in-line monitoring of the freeze-drying process using an NIR spectroscopy tool. J. Pharm. Sci. 107, 139-148, doi:10.1016/j.xphs.2017.05.024 (2018).

41. Miller, D. P. \& de Pablo, J. J. Calorimetric solution properties of simple saccharides and their significance for the stabilization of biological structure and function. J. Phys. Chem. B 104, 88768883 (2000).

42. Suga, K. \& Umakoshi, H. Detection of nanosized ordered domains in DOPC/DPPC and DOPC/Ch binary lipid mixture systems of large unilamellar vesicles using a TEMPO quenching method. Langmuir 29, 4830-4838, doi:10.1021/la304768f (2013).

43. Han, J., Suga, K., Hayashi, K., Okamoto, Y. \& Umakoshi, H. Multi-level characterization of the membrane properties of resveratrol-incorporated liposomes. J. Phys. Chem. B 121, 4091-4098, doi:10.1021/acs.jpcb.7b00368 (2017).

\section{Figures}


a

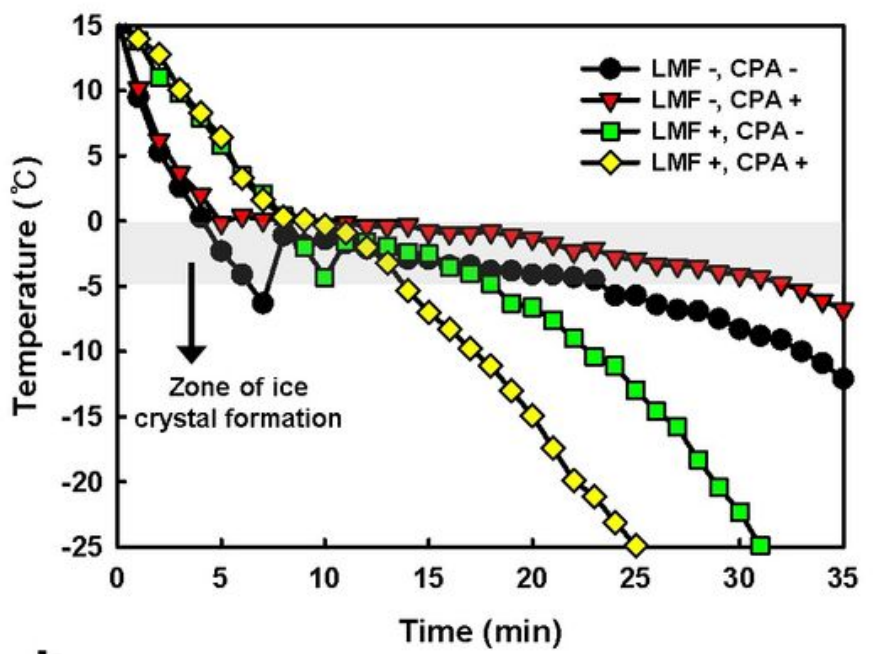

b

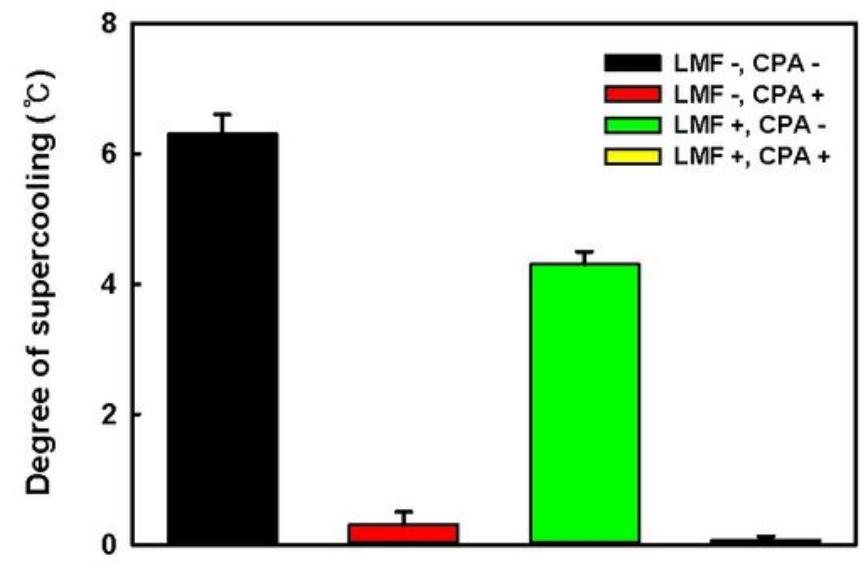

C

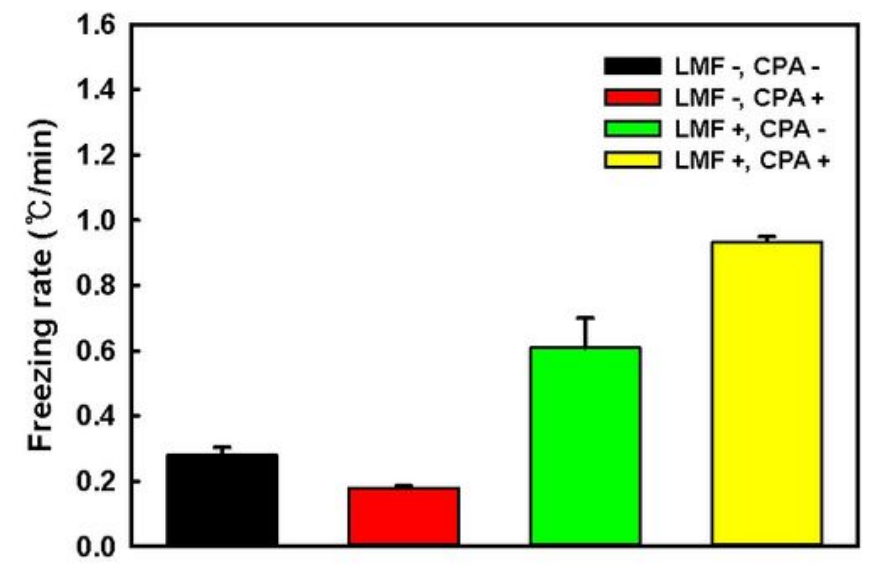

Figure 1

Effects of LMF and CPA on the freezing behavior of the lipid nanovesicle solution. (a) Temperature profiles, (b) degree of supercooling, and (c) freezing rate under different freezing conditions. Isopropanol was used as the liquid medium for the LMF system. The freezing rate was measured within the zone of ice crystal formation $\left(-5 \sim 0{ }^{\circ} \mathrm{C}\right)$. 

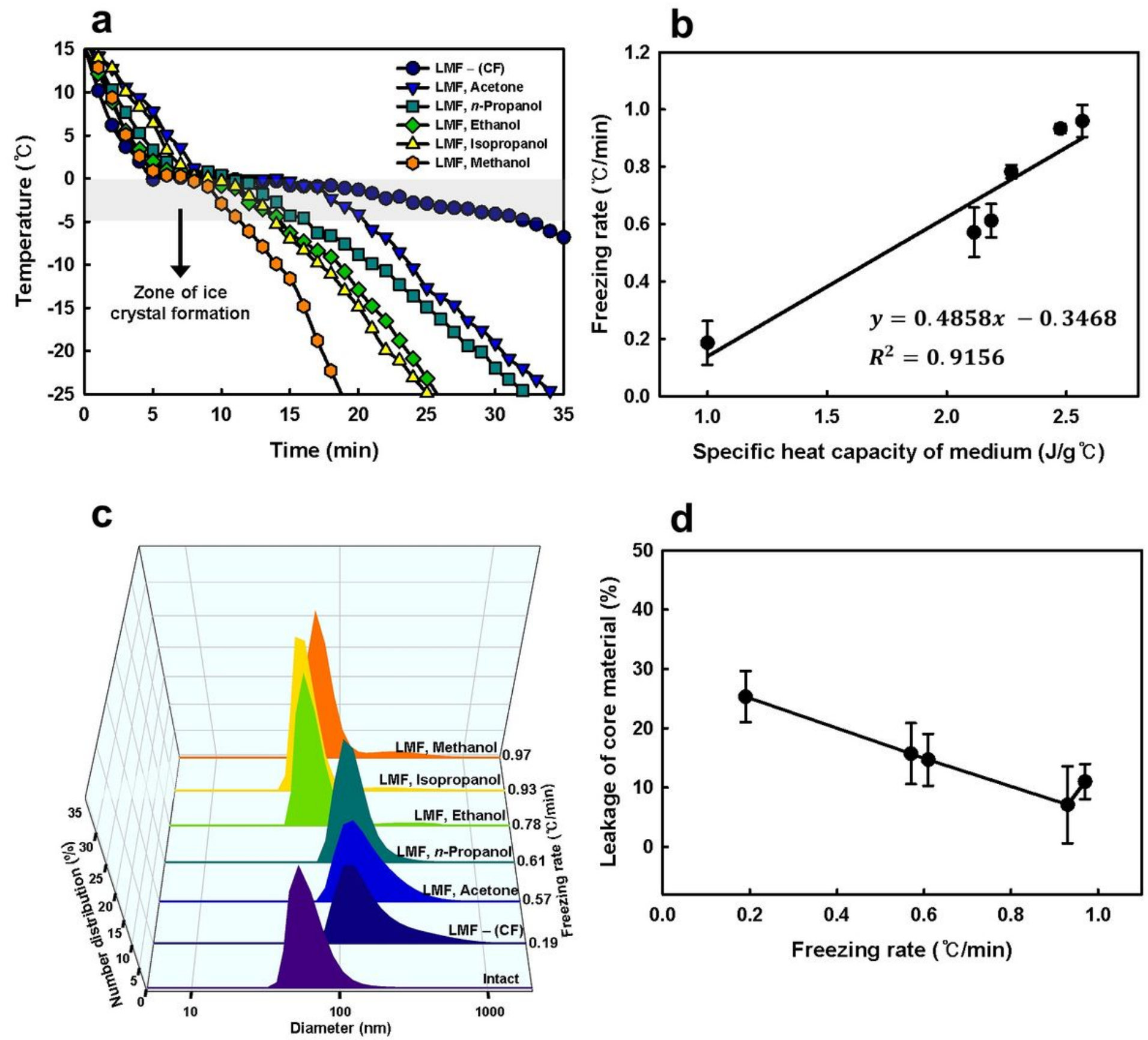

Figure 2

Effects of the medium (organic solvent) in the LMF system on the freezing rate and physicochemical properties of lipid nanovesicles. (a) Temperature profiles of lipid nanovesicles in the CF and LMF systems with different media. (b) Correlation between the specific heat capacity of the medium and freezing rate of lipid nanovesicles. (c) Size distribution and (d) leakage of encapsulated material (fluorescent calcein) of lyophilized lipid nanovesicles in the various freezing systems with different freezing rates. The size distribution and leakage of lyophilized lipid nanovesicles were measured after rehydration. 
a

Lipid nanovesicle powder

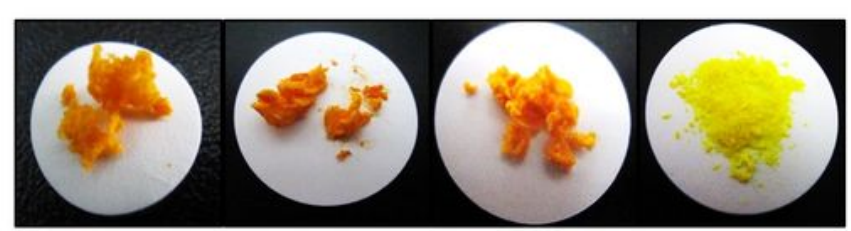

Rehydrated lipid nanovesicle

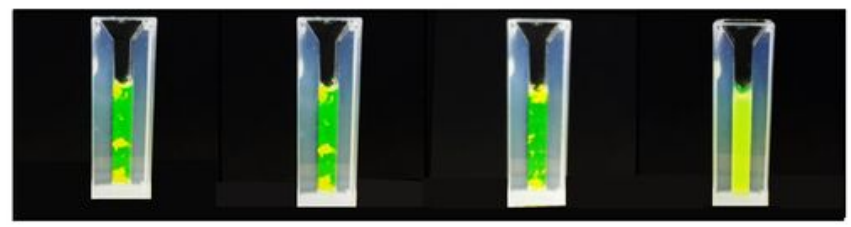

b
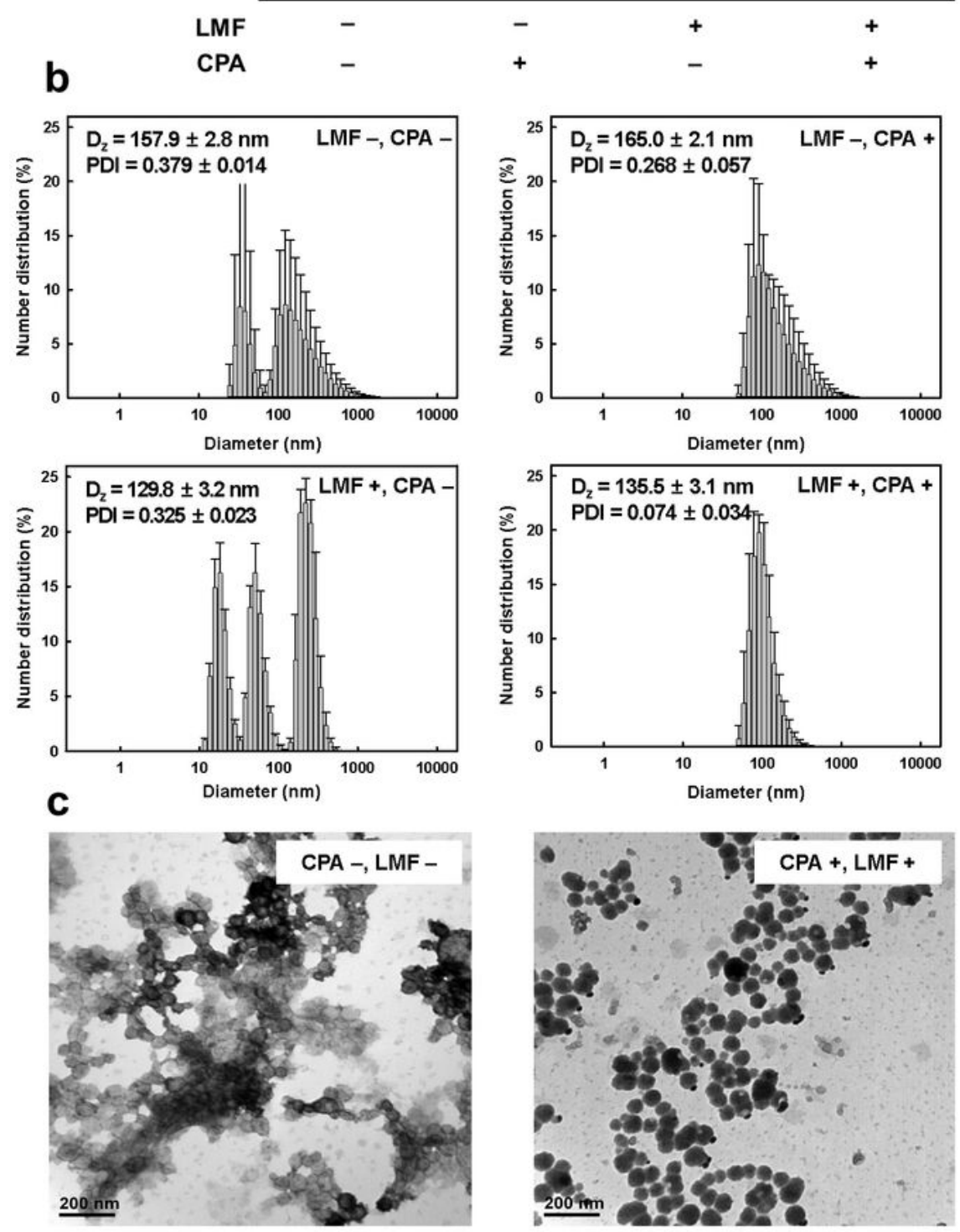

Figure 3

Effects of LMF and CPA on the rehydration behavior of lipid nanovesicle powders produced by lyophilization. (a) Appearance of lipid nanovesicle powders and rehydrated lipid nanovesicles. (b) Size distribution and (c) TEM images of lipid nanovesicle powders after rehydration. 


\section{a}
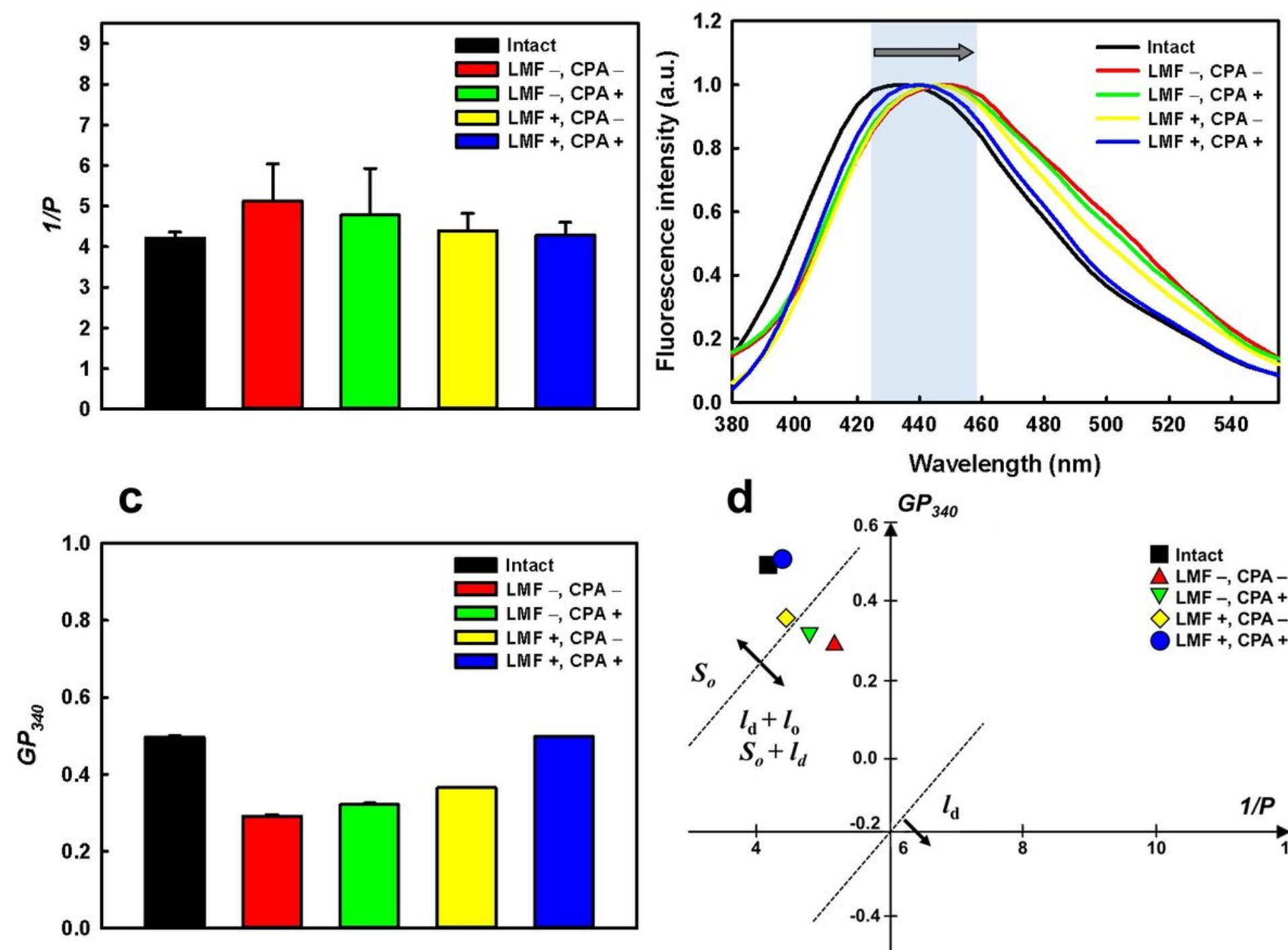

b

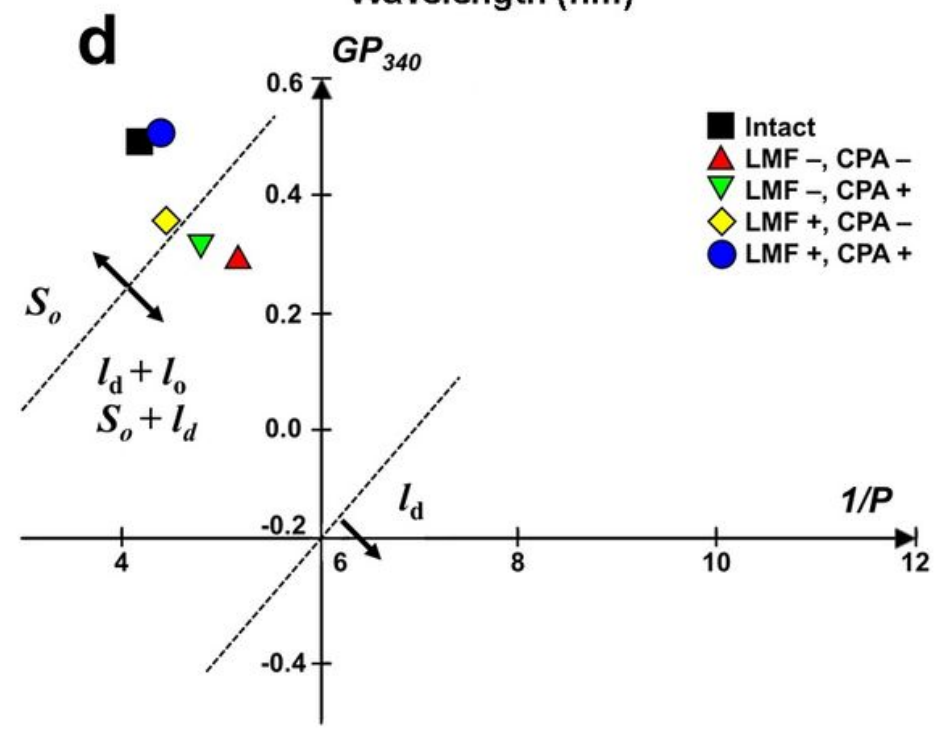

\section{Figure 4}

Membrane fluidity and polarity of intact lipid nanovesicles and lyophilized lipid nanovesicles with different freezing conditions, after rehydration. (a) The membrane fluidity $(1 / P)$ values of the lipid nanovesicles measured using 1,6-diphenyl-1,3,5-hexatriene fluorescent dye. (b) Fluorescence spectra of Laurdan intensity and (c) the membrane polarity (GP340) values of the lipid nanovesicles. Total concentrations of lipid and Laurdan in the test solution were 200 and $1 \mu \mathrm{M}$, respectively. (d) Cartesian diagram of the lipid nanovesicles. The $x$ and $y$ axes indicate 1/P and GP340 values, respectively. 


\section{Lyophilization Rehydration}

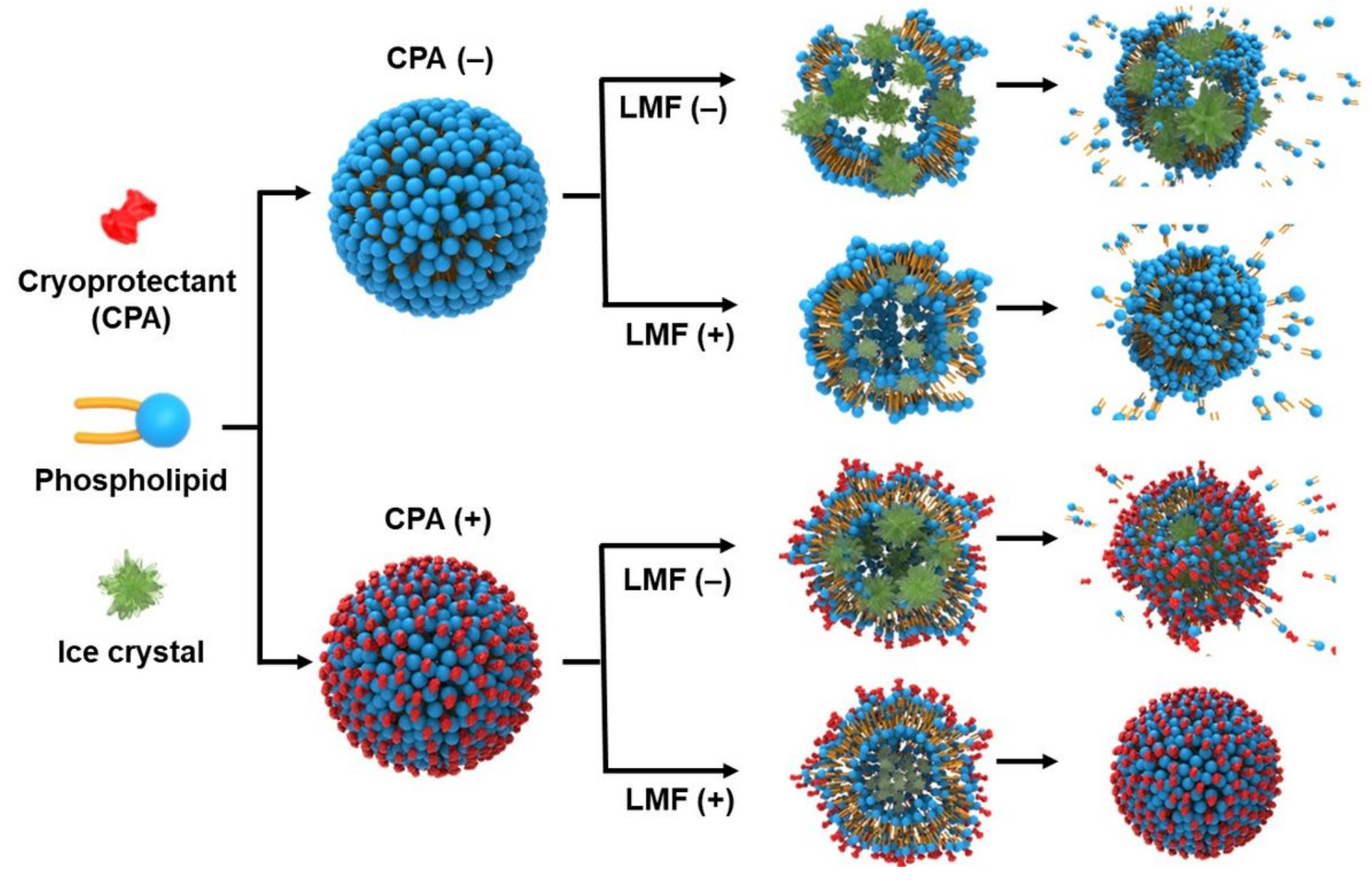

Figure 5

Schematic illustration representing the effects of CPA and LMF on lipid bilayer membranes during lyophilization and rehydration. 

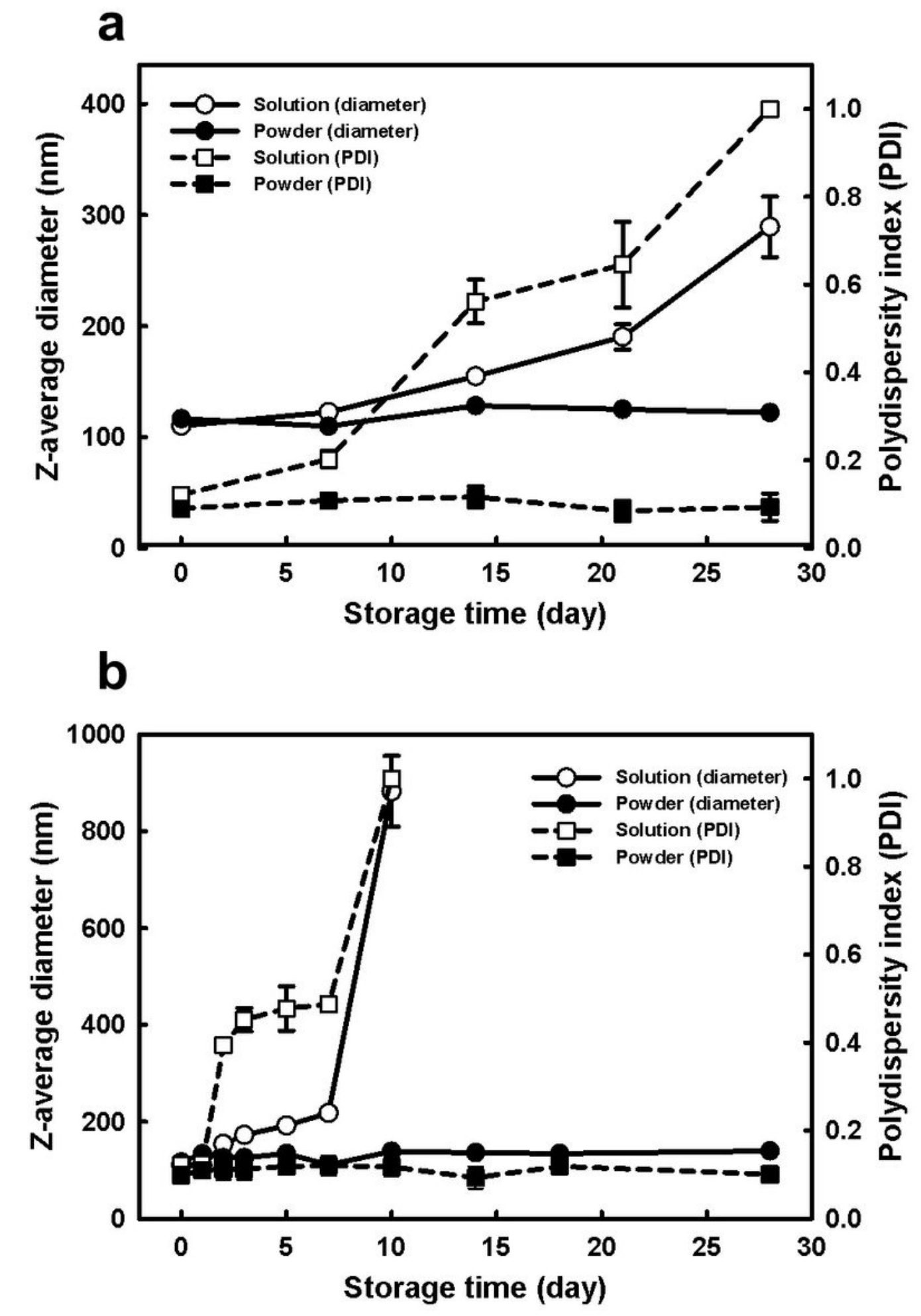

Figure 6

Storage stability of lipid nanovesicle solutions and lyophilized lipid nanovesicle powders incubated at (a) $4{ }^{\circ} \mathrm{C}$ and (b) $30^{\circ} \mathrm{C}$. The lyophilized sample was produced via lyophilization with cryoprotectant (CPA) and liquid-mediated freezing (LMF) using isopropanol. Diameter, Z-average diameter; PDI, polydispersity index. 


\section{Supplementary Files}

This is a list of supplementary files associated with this preprint. Click to download.

- Supplementaryfile.docx 